\title{
Note on an inequality
}

\author{
Yongzhong $\mathrm{Xu}$ \\ Rutgers University, Department of Mathematics, 110 Frelinghuysen Road, Piscataway, NJ 08854-8019, USA
}

Received 26 January 2005; received in revised form 26 May 2005; accepted 5 July 2005

Available online 9 December 2005

\begin{abstract}
We prove in this article the case of three masses, of an inequality of discrete type (which might have a continuous extension) which is still a conjecture for any $p$ points in $\mathbb{R}^{3}$. The inequality appears naturally in the derivation of Morse Lemma at infinity for Yamabe problems with changing signs. We also explain why this inequality might hold in general.

(c) 2006 L'Association Publications de l'Institut Henri Poincaré. Published by Elsevier B.V. All rights reserved
\end{abstract}

\section{Résumé}

Nous prouvons dans cet article le cas $p=3$, d'une inégalité discrète (qui s'étend peut-être au cas continu) qui est une conjecture pour $p$ points quelconques de $\mathbb{R}^{3}$. Cette inégalité apparaît naturellement dans la démonstration du Lemme de Morse à l'infini [A. Bahri, Critical Points at Infinity in Some Variational Problems, Pitman Res. Notes Math. Ser., vol. 182, Longman Scientific \& Technical, Harlow, 1989] pour les problèmes de Yamabe avec changement de signe. Nous montrons par la suite pourquoi l'inégalité devrait être vraie en général.

(C) 2006 L'Association Publications de l'Institut Henri Poincaré. Published by Elsevier B.V. All rights reserved

Keywords: Inequality of discrete type; Yamabe problem; Changing-sign solution; Morse lemma at infinity

\section{Introduction}

The aim of this note is to prove the case of three masses, of an inequality which is conjectured in [8] and used in order to establish a Morse lemma at infinity in the changing sign Yamabe problem on $S^{3}$.

Let $A=\left(a_{i j}\right)$ be the $p \times p$ matrix, with $a_{i i}=0$, and $a_{i j}=1 /\left|x_{i}-x_{j}\right|$, for $1 \leqslant i, j \leqslant p$. The conjecture reads as follows:

Conjecture 1. There exists $c(p)>0$, such that, for any $\left(x_{1}, \ldots, x_{p}\right) \in \mathbb{R}^{3 p}$, and for any $u \in \mathbb{R}^{p}$,

$$
\sup _{1 \leqslant i \leqslant p}\left|{ }^{t} u\left[\frac{\partial A}{\partial x_{i}}\right] u\right|+|A u|^{2} \geqslant c \sum_{i \neq j} \frac{u_{i}^{2}}{\left|x_{i}-x_{j}\right|^{2}} .
$$

E-mail addresses: xuyz@math.rutgers.edu, abbyxu@gmail.com (Y. Xu). 
The inequality might seem somewhat surprising, but it arises in a natural way when one tries to establish a Morse lemma at infinity for the Yamabe changing-sign problem on $S^{3}$, see [5,8]. We would like to explain briefly in the introduction how it arises.

Let $\left(S^{3}, c\right)$ be $S^{3}$ equipped with the standard metric and let $J(u)=1 / \int_{S^{3}} u^{6} \mathrm{~d} v$ be the Yamabe functional defined on $\Sigma=\left\{u\right.$ such that $\left.\int\left(\left|\nabla_{S^{3}} u\right|^{2}+\frac{3}{4} u^{2}\right) \mathrm{d} v=1\right\}$. Critical points for $J$ are known to exist, in fact infinitely many critical points are known to exist. Because of the non-compactness of the conformal group, they concentrate and combine to build asymptotes, see [1-3,6]. The difference of topology at the level set of $J$ induced by these asymptotes has never been computed. Hence, one can say there is a variational problem where several critical points are known, but the variational problem is not understood.

Consider a family of solutions $\overline{\omega_{1}}, \ldots, \overline{\omega_{p}}$, one can combine them into $\sum_{i=1}^{p} \sqrt{\lambda_{i}} \overline{\omega_{i}}\left(\lambda_{i}\left(x-a_{i}\right)\right)$ after stereographic projection on $\mathbb{R}^{3}$. If the $a_{i}$ 's remain in a compact set and the $\lambda_{i}$ 's tend to $+\infty$ and if

$$
\varepsilon_{i j}=1 /\left(\frac{\lambda_{i}}{\lambda_{j}}+\frac{\lambda_{j}}{\lambda_{i}}+\lambda_{i} \lambda_{j}\left|a_{i}-a_{j}\right|^{2}\right)^{1 / 2}
$$

tends to zero, then $J^{\prime}\left(\sum_{i=1}^{p} \sqrt{\lambda_{i}} \overline{\omega_{i}}\left(\lambda_{i}\left(x-a_{i}\right)\right)\right)$ tends to zero, i.e. $\sum_{i=1}^{p} \sqrt{\lambda_{i}} \overline{\omega_{i}}\left(\lambda_{i}\left(x-a_{i}\right)\right)$ builds an asymptote.

A good parametrization of a neighborhood of this asymptote is provided by

$$
u=\sum_{i=1}^{p} \alpha_{i} \sqrt{\lambda_{i}} \overline{\omega_{i}}\left(\lambda_{i}\left(x-a_{i}\right)\right)+v=\sum_{i=1}^{p} \alpha_{i} \omega_{i}+v,
$$

where $v$ is small and satisfies a family of orthogonality conditions [2,5,8].

Expanding $J(u)$, we find

$$
J(u)=\frac{\left(\sum_{i=1}^{p} \alpha_{i}^{2} \int{\overline{\omega_{i}}}^{6}\right)^{3}}{\sum_{i=1}^{p} \alpha_{i}^{6} \int \bar{\omega}_{i}^{6}}(1+P+R+(f, v)+Q(v, v)) .
$$

Here $P$ is the principal term in the expansion,

$$
P=\sum_{i \neq j}\left(\overline{\omega_{i}}\left(\tilde{a}_{j}\right){\overline{\omega_{j}}}^{\infty}-c_{i j} \varepsilon_{i j}^{3}\right),
$$

where $\overline{\omega_{j}} \infty$ is the value of $\overline{\omega_{j}}$ at the north pole (with $\sqrt{\lambda_{j}} \overline{\omega_{j}}\left(\lambda_{j}\left(x-a_{j}\right)\right)$ concentrated at the south pole) and $\overline{\omega_{i}}\left(\tilde{a}_{j}\right)$ are the value of $\overline{\omega_{i}}$ at the new concentration point of $\omega_{j}$ after re-scaling $\overline{\omega_{i}}$ to concentration 1 . And the reminder term $R$ reads:

$$
R=\mathrm{o}\left(\sum\left(\left(\overline{\omega_{i}}{ }^{\infty 2}+\overline{\omega_{j}}\left(\widetilde{a_{j}}\right)^{2}\right) \varepsilon_{i j}^{2}+\varepsilon_{i j}^{3}\right)+|v|_{H^{1}}^{2}\right) .
$$

Under minimal assumptions of non-degeneracy (i.e.transversallity to their invariance group) of the $\omega_{j}$ 's, $(f, v)+$ $Q(v, v)+\mathrm{o}\left(|v|_{H^{1}}^{2}\right)$ can be extremized. Thus we have derived a new

$$
\bar{u}=\sum_{i=1}^{p} \alpha_{i} \omega_{i}+\bar{v}
$$

and

$$
J(u)=J(\bar{u})+Q(v, v),
$$

where $v$ is a new small linear parameter standing for $v-\bar{v}$ (or so).

Then $J(\bar{u})$ reads basically as

$$
J(\bar{u})=\frac{\left(\sum_{i=1}^{p} \alpha_{i}^{2} \int{\overline{\omega_{i}}}^{6}\right)^{3}}{\sum_{i=1}^{p} \alpha_{i}^{6} \int \bar{\omega}_{i}^{6}}\left(1+P_{1}+R_{1}\right),
$$

where $P_{1}$ and $R_{1}$ behave exactly as $P$ and $R$, so we drop the subscript 1 in what follows.

The Morse lemma at infinity then reads (it is still a conjecture if no assumption is introduced at this time, see [8]): 
Morse lemma at infinity. There exists a change of coordinates in the $\left(a_{i}, \lambda_{i}\right)$ spaces, $\left(a_{i}, \lambda_{i}\right) \rightarrow\left(\widetilde{a_{i}}, \tilde{\lambda_{i}}\right)$ such that $J(u)$ reads as

$$
J(\bar{u})=\frac{\left(\sum_{i=1}^{p} \alpha_{i}^{2} \int{\overline{\omega_{i}}}^{6}\right)^{3}}{\sum_{i=1}^{p} \alpha_{i}^{6} \int \bar{\omega}_{i}^{6}}\left(1+\widetilde{P}_{1}^{\prime}\right),
$$

where $\widetilde{P}_{1}^{\prime}$ is $P_{1}$ with the variables $a_{i}^{\prime}, \lambda_{i}^{\prime}, \lambda_{j}$.

We now provide a sketch of the proof of this lemma under more assumptions [8]. This will show how our inequality enters into play.

$\bar{u}$ contains only the variables $\lambda_{i}, \alpha_{i}, a_{i}, \sigma_{i}$. In order to complete a Morse lemma at infinity, we need to estimate

$$
\begin{aligned}
& \text { (i) } \frac{\partial J(\bar{u})}{\partial \alpha_{i}}=J^{\prime}(\bar{u}) \cdot\left(\overline{\omega_{i}}+\frac{\partial \bar{v}}{\partial \alpha_{i}}\right), \\
& \text { (ii) } \lambda_{i} \frac{\partial J(\bar{u})}{\partial \lambda_{i}}=J^{\prime}(\bar{u}) \cdot\left(\lambda_{i} \frac{\partial \overline{\omega_{i}}}{\partial \lambda_{i}}+\lambda_{i} \frac{\partial \bar{v}}{\partial \lambda_{i}}\right), \\
& \text { (iii) } \frac{\partial J(\bar{u})}{\lambda_{i} \partial a_{i}}=J^{\prime}(\bar{u}) \cdot\left(\frac{\partial \overline{\omega_{i}}}{\lambda_{i} \partial a_{i}}+\frac{\partial \bar{v}}{\lambda_{i} \partial a_{i}}\right), \\
& \text { (iv) } \frac{\partial J(\bar{u})}{\partial \sigma_{i}}=J^{\prime}(\bar{u}) \cdot\left(\frac{\partial \overline{\omega_{i}}}{\partial \sigma_{i}}+\frac{\partial \bar{v}}{\partial \sigma_{i}}\right) .
\end{aligned}
$$

The $\bar{v}$ derivatives can be easily handled using a trick involving the orthogonality relations satisfied by $\bar{v}$.

When we are dealing with positive solutions, the $\overline{\omega_{i}}$ 's are equal to the $\delta_{i}$ 's. In this case, one can easily see that (i), (ii) and (iii) work together by taking derivatives of $P$. Indeed $\overline{\omega_{i}}\left(\widetilde{a_{j}}\right)$ and $\overline{\omega_{j}} \infty$ are constants equal to $c_{0}>0$. The $\lambda_{i}$-derivatives work together and provide estimates. They do not destroy each other. This basic fact helps in order to build a pseudo-gradient out of (i)-(iii).

When the positivity assumption is dropped, these estimates are lost and we need large variations in the 'compact' variables, which are all the variables besides the $\lambda_{i}$ 's (the $a_{i}$ 's live on $S^{3}$ ).

We are then led to estimate $\partial J(\bar{u}) / \partial \alpha_{i}$ in lieu of $\partial J(\bar{u}) / \lambda_{i} \partial a_{i}$.

Computing $\partial P / \partial a_{i}$, under the assumption that $\varepsilon_{i j}=1 /\left(\sqrt{\lambda_{i} \lambda_{j}}\left|a_{i}-a_{j}\right|\right)$, we find (identifying $\overline{\omega_{i}}\left(\widetilde{a_{j}}\right)$ and $\overline{\omega_{j}} \infty$ for the sake of simplicity)

$$
-\sum_{i \neq j} \bar{\omega}_{i}^{\infty} \bar{\omega}_{j}^{\infty} \frac{a_{i}-a_{j}}{\sqrt{\lambda_{i} \lambda_{j}}\left|a_{i}-a_{j}\right|^{3}}+c_{i j} 3 \sum_{i \neq j} \varepsilon_{i j}^{3} \frac{a_{i}-a_{j}}{\left|a_{i}-a_{j}\right|^{2}} .
$$

The first term can be identified as

$$
\left(\ldots \frac{\bar{\omega}_{i} \infty}{\sqrt{\lambda_{i}}} \ldots\right) \frac{\partial A}{\partial a_{i}}\left(\ldots \frac{\bar{\omega}_{i}^{\infty}}{\sqrt{\lambda_{i}}} \ldots\right)^{t}
$$

while the second term is

$$
\mathrm{O}\left(\sum_{i \neq j} \sqrt{\lambda_{i} \lambda_{j}} \varepsilon_{i j}^{4}\right)
$$

Continuing a thorough and difficult computation, we find that the derivatives of the remainder term $R$ behave as

$$
\left|\frac{\partial R}{\partial a_{i}}\right|=\mathrm{o}\left(\sum_{j \neq l} \lambda_{l}{\overline{\omega_{j}}}^{\infty 2} \varepsilon_{j l}^{2}+\sum_{j \neq l} \sqrt{\lambda_{j} \lambda_{l}}, \varepsilon_{j l}^{4}\right) .
$$

Actually, the estimate is much better because $\varepsilon_{i j}$ is a factor in $\partial R / \partial a_{i}$ (a square root of it depends only on $i$ ). Work is under progress to prove that, in this statement, we can take $j=i$.

On the other hand,

$$
-2 \lambda_{i} \frac{\partial P}{\partial \lambda_{i}}=\sum \bar{\omega}_{i}^{\infty} \sum_{i \neq j} \bar{\omega}_{j}^{\infty} \frac{1}{\sqrt{\lambda_{i} \lambda_{j}}\left|a_{i}-a_{j}\right|}+\mathrm{O}\left(\varepsilon_{i j}^{3}\right)
$$


while

$$
\frac{\partial P}{\partial \sigma_{i}}=\frac{\partial \bar{\omega}_{i}^{\infty}}{\partial \sigma_{i}} \sum_{i \neq j} \bar{\omega}_{j}^{\infty} \frac{1}{\sqrt{\lambda_{i} \lambda_{j}}\left|a_{i}-a_{j}\right|}+\mathrm{O}\left(\varepsilon_{i j}^{3}\right) .
$$

Assuming that

$$
\left|\bar{\omega}_{i}^{\infty}\right|+\left|\frac{\partial \bar{\omega}_{i}^{\infty}}{\partial \sigma_{i}}\right| \geqslant c>0,
$$

we derive:

$$
\sum_{i=1}^{p}\left|\lambda_{i} \frac{\partial P}{\partial \lambda_{i}}\right|+\left|\frac{\partial P}{\partial \sigma_{i}}\right| \geqslant|A u|+\mathrm{O}\left(\sum \varepsilon_{i j}^{3}\right) .
$$

Combining with the $a_{i}$ 's and these derivatives, we rebuild

$$
\sup _{i}\left|{ }^{t} u\left[\frac{\partial A}{\partial x_{i}}\right] u\right|+|A u|^{2} \text {. }
$$

We want the above term to be much larger than the derivatives of the remainder term in the expansion of $J$. Comparing, we reach our inequality.

As in [5], the Morse lemma at infinity is established in [8] when the $\lambda_{i}$ 's satisfy

$$
\frac{1}{c} \leqslant \frac{\lambda_{i}}{\lambda_{j}} \leqslant c
$$

with $c$ a fixed constant. However the expansion is general and we expect this hypothesis to be removed soon. Our inequality becomes crucial in this process.

This inequality is difficult to establish. We proved for the case of $p=3$. Although this seems to be quite limited, the application is in fact large since it establishes the Morse lemma at infinity for all possible triplet $\left(\overline{\omega_{1}}, \overline{\omega_{2}}, \overline{\omega_{3}}\right)$ of solutions of the Yamabe changing-sign problem on $S^{3}$. We expect of course this Morse lemma at infinity and the techniques of $[1,4,7]$ to extend to Yamabe-type problems.

Thus our theorem reads:

Theorem 1. There exists a constant $c_{3}>0$, such that for every $\left(x_{1}, x_{2}, x_{3}\right) \in \mathbb{R}^{9}$ and $\left(u_{1}, u_{2}, u_{3}\right) \in \mathbb{R}^{3}$,

$$
\sup _{1 \leqslant i \leqslant 3}\left|t u\left[\frac{\partial A}{\partial x_{i}}\right] u\right|+|A u|^{2} \geqslant c_{3} \sum_{i \neq j} \frac{u_{i}^{2}}{\left|x_{i}-x_{j}\right|^{2}} .
$$

The remaining part of this paper is devoted to the proof of this theorem.

The proof is completed by carefully examining for the relative positions of the $x_{i}$ 's. We denote

$$
a=\left|x_{2}-x_{3}\right|, \quad b=\left|x_{1}-x_{3}\right|, \quad c=\left|x_{1}-x_{2}\right| .
$$

Without loss of generality, we can assume $a \geqslant b \geqslant c$, therefore $\theta_{1} \geqslant \theta_{2} \geqslant \theta_{3}$.

We discuss three distinct cases:

Case I, the lengths of $a, b$ and $c$ are comparable, and the three angles $\theta_{1}, \theta_{2}$ and $\theta_{3}$ are neither very small nor very close to $\pi$. In this case, the first term of the left-hand side of the inequality is able to balance the second term, the proof is quite straightforward;

Case II, $c$ is very small compared with $a$. In this case, we prove the inequality by looking at the minimization problem

$$
\operatorname{Min} \frac{\left|u_{3}\left(u_{1} / b^{2}+\left(u_{2} / a^{2}\right) \cos \theta_{3}\right)\right|+u_{1} u_{2} /(a b)+u_{1} u_{3} /(a c)+u_{2} u_{3} /(b c)}{\left(u_{1}^{2}+u_{2}^{2}\right) / c^{2}+\left(u_{2}^{2}+u_{3}^{2}\right) / a^{2}+\left(u_{1}^{2}+u_{3}^{2}\right) / b^{2}}=J\left(u_{1}, u_{2}, u_{3}\right) ;
$$

Case III, we prove all the remaining cases by carefully balancing the two terms directly. 


\section{Details of the proof}

For $p=3$,

$$
{ }^{t} u\left[\frac{\partial A}{\partial x_{1}}\right] u=2\left|u_{1}\right| \sqrt{\frac{u_{2}^{2}}{\left|x_{1}-x_{2}\right|^{4}}+\frac{u_{3}^{2}}{\left|x_{1}-x_{3}\right|^{4}}+2 u_{2} u_{3} \frac{\left(x_{1}-x_{2}, x_{1}-x_{3}\right)}{\left|x_{1}-x_{2}\right|^{3}\left|x_{1}-x_{3}\right|^{3}}} .
$$

Similarly we have

$$
\begin{aligned}
& { }^{t} u\left[\frac{\partial A}{\partial x_{2}}\right] u=2\left|u_{2}\right| \sqrt{\frac{u_{1}^{2}}{\left|x_{2}-x_{1}\right|^{4}}+\frac{u_{3}^{2}}{\left|x_{2}-x_{3}\right|^{4}}+2 u_{1} u_{3} \frac{\left(x_{2}-x_{1}, x_{2}-x_{3}\right)}{\left|x_{2}-x_{1}\right|^{3}\left|x_{3}-x_{2}\right|^{3}}}, \\
& { }^{t} u\left[\frac{\partial A}{\partial x_{3}}\right] u=2\left|u_{3}\right| \sqrt{\frac{u_{1}^{2}}{\left|x_{3}-x_{1}\right|^{4}}+\frac{u_{2}^{2}}{\left|x_{3}-x_{2}\right|^{4}}+2 u_{1} u_{2} \frac{\left(x_{3}-x_{1}, x_{3}-x_{2}\right)}{\left|x_{3}-x_{1}\right|^{3}\left|x_{3}-x_{2}\right|^{3}},}
\end{aligned}
$$

and

$$
\begin{aligned}
|A u|^{2}= & \left\{\frac{u_{1}^{2}+u_{2}^{2}}{\left|x_{1}-x_{2}\right|^{2}}+\frac{u_{2}^{2}+u_{3}^{2}}{\left|x_{3}-x_{2}\right|^{2}}+\frac{u_{1}^{2}+u_{3}^{2}}{\left|x_{1}-x_{3}\right|^{2}}\right\} \\
& +2\left\{\frac{u_{1} u_{2}}{\left|x_{3}-x_{1}\right|\left|x_{3}-x_{2}\right|}+\frac{u_{1} u_{3}}{\left|x_{2}-x_{1}\right|\left|x_{2}-x_{3}\right|}+\frac{u_{2} u_{3}}{\left|x_{1}-x_{2}\right|\left|x_{1}-x_{3}\right|}\right\} .
\end{aligned}
$$

Therefore in order to establish our theorem for $p=3$ we need to prove that there exists a constant $c$ such that

$$
\begin{aligned}
& \sup \left\{\begin{array}{l}
2\left|u_{1}\right| \sqrt{\frac{u_{2}^{2}}{\left|x_{1}-x_{2}\right|^{4}}+\frac{u_{3}^{2}}{\left|x_{1}-x_{3}\right|^{4}}+2 u_{2} u_{3} \frac{\left(x_{1}-x_{2}, x_{1}-x_{3}\right)}{\left|x_{1}-x_{2}\right|^{3}\left|x_{1}-x_{3}\right|^{3}}} \\
2\left|u_{2}\right| \sqrt{\frac{u_{1}^{2}}{\left|x_{2}-x_{1}\right|^{4}}+\frac{u_{3}^{2}}{\left|x_{2}-x_{3}\right|^{4}}+2 u_{1} u_{3} \frac{\left(x_{2}-x_{1}, x_{2}-x_{3}\right)}{\left|x_{2}-x_{1}\right|^{3}\left|x_{3}-x_{2}\right|^{3}}} \\
2\left|u_{3}\right| \sqrt{\frac{u_{1}^{2}}{\left|x_{3}-x_{1}\right|^{4}}+\frac{u_{2}^{2}}{\left|x_{3}-x_{2}\right|^{4}}+2 u_{1} u_{2} \frac{\left(x_{3}-x_{1}, x_{3}-x_{2}\right)}{\left|x_{3}-x_{1}\right|^{3}\left|x_{3}-x_{2}\right|^{3}}}
\end{array}\right\} \\
& +\left\{\frac{u_{1}^{2}+u_{2}^{2}}{\left|x_{1}-x_{2}\right|^{2}}+\frac{u_{2}^{2}+u_{3}^{2}}{\left|x_{3}-x_{2}\right|^{2}}+\frac{u_{1}^{2}+u_{3}^{2}}{\left|x_{1}-x_{3}\right|^{2}}\right\} \\
& +2\left\{\frac{u_{1} u_{2}}{\left|x_{3}-x_{1}\right|\left|x_{3}-x_{2}\right|}+\frac{u_{1} u_{3}}{\left|x_{2}-x_{1}\right|\left|x_{2}-x_{3}\right|}+\frac{u_{2} u_{3}}{\left|x_{1}-x_{2}\right|\left|x_{1}-x_{3}\right|}\right\} \\
& \geqslant c\left\{\frac{u_{1}^{2}+u_{2}^{2}}{\left|x_{1}-x_{2}\right|^{2}}+\frac{u_{2}^{2}+u_{3}^{2}}{\left|x_{3}-x_{2}\right|^{2}}+\frac{u_{1}^{2}+u_{3}^{2}}{\left|x_{1}-x_{3}\right|^{2}}\right\}
\end{aligned}
$$

for any $x_{i} \in \mathbb{R}^{3}, u_{i} \in R, i=1,2,3$.

Claim 1. We have:

$$
\begin{aligned}
& \left|u_{1}\right| \sqrt{\frac{u_{2}^{2}}{c^{4}}+\frac{u_{3}^{2}}{b^{4}}+2 u_{2} u_{3} \frac{\cos \theta_{1}}{b^{2} c^{2}}} \\
& \geqslant \sup \left\{\frac{\left|u_{1} u_{3}\right| \sin \theta_{1}}{b^{2}}, \frac{\left|u_{1} u_{2}\right| \sin \theta_{1}}{c^{2}},\left|u_{1}\right|\left|\frac{u_{2}}{c^{2}}+\frac{u_{3}}{b^{2}} \cos \theta_{1}\right|,\left|u_{1}\right|\left|\frac{u_{2}}{c^{2}} \cos \theta_{1}+\frac{u_{3}}{b^{2}}\right|\right\}, \\
& \left|u_{2}\right| \sqrt{\frac{u_{1}^{2}}{c^{4}}+\frac{u_{3}^{2}}{a^{4}}+2 u_{1} u_{3} \frac{\cos \theta_{2}}{a^{2} c^{2}}} \\
& \geqslant \sup \left\{\frac{\left|u_{1} u_{2}\right| \sin \theta_{2}}{c^{2}}, \frac{\left|u_{2} u_{3}\right| \sin \theta_{2}}{a^{2}},\left|u_{2}\right|\left|\frac{u_{1}}{c^{2}}+\frac{u_{3}}{a^{2}} \cos \theta_{2}\right|,\left|u_{1}\right|\left|\frac{u_{1}}{c^{2}} \cos \theta_{2}+\frac{u_{3}}{a^{2}}\right|\right\},
\end{aligned}
$$


and

$$
\begin{aligned}
& \left|u_{3}\right| \sqrt{\frac{u_{1}^{2}}{b^{4}}+\frac{u_{2}^{2}}{a^{4}}+2 u_{1} u_{2} \frac{\cos \theta_{3}}{a^{2} b^{2}}} \\
& \geqslant \sup \left\{\frac{\left|u_{1} u_{3}\right| \sin \theta_{3}}{b^{2}}, \frac{\left|u_{2} u_{3}\right| \sin \theta_{3}}{c^{2}},\left|u_{3}\right|\left|\frac{u_{1}}{b^{2}}+\frac{u_{2}}{a^{2}} \cos \theta_{3}\right|,\left|u_{3}\right|\left|\frac{u_{1}}{b^{2}} \cos \theta_{3}+\frac{u_{2}}{a^{2}}\right|\right\} .
\end{aligned}
$$

Proof of Claim 1. It is easy to see that

$$
\sqrt{\frac{u_{2}^{2}}{c^{4}}+\frac{u_{3}^{2}}{b^{4}}+2 u_{2} u_{3} \frac{\cos \theta_{1}}{b^{2} c^{2}}}=\sqrt{\left(\frac{u_{2}}{c^{2}}+\frac{u_{3}}{b^{2}} \cos \theta_{1}\right)^{2}+\frac{u_{3}^{2} \sin ^{2} \theta_{1}}{b^{4}}} \geqslant \sup \left\{\left|\frac{u_{2}}{c^{2}}+\frac{u_{3}}{b^{2}} \cos \theta_{1}\right|, \frac{\left|u_{3}\right| \sin \theta_{1}}{b^{2}}\right\},
$$

and

$$
\sqrt{\frac{u_{2}^{2}}{c^{4}}+\frac{u_{3}^{2}}{b^{4}}+2 u_{2} u_{3} \frac{\cos \theta_{1}}{b^{2} c^{2}}}=\sqrt{\left(\frac{u_{3}}{b^{2}}+\frac{u_{2}}{c^{2}} \cos \theta_{1}\right)^{2}+\frac{u_{2}^{2} \sin ^{2} \theta_{1}}{c^{4}}} \geqslant \sup \left\{\left|\frac{u_{3}}{b^{2}}+\frac{u_{2}}{c^{2}} \cos \theta_{1}\right|, \frac{\left|u_{2}\right| \sin \theta_{1}}{c^{2}}\right\} .
$$

Similarly, we can prove the remaining two inequalities.

We would like to compare these expressions with

$$
\frac{u_{1} u_{2}}{a b}+\frac{u_{1} u_{3}}{a c}+\frac{u_{2} u_{3}}{b c} \text { and } \frac{u_{1}^{2}+u_{2}^{2}}{c^{2}}+\frac{u_{2}^{2}+u_{3}^{2}}{a^{2}}+\frac{u_{1}^{2}+u_{3}^{2}}{b^{2}} \text {. }
$$

Case I: the lengths of $a, b$ and $c$ satisfy $b+c-a \geqslant a / 100$. Now we look at the case that $b+c-a \geqslant a / 100$. It is easy to see that

$$
\begin{aligned}
\left(\frac{\left|u_{1} u_{3}\right| \sin \theta_{3}}{b^{2}}\right)^{2} /\left(\frac{u_{1} u_{3}}{a c}\right)^{2} & =\frac{a^{2} c^{2}\left(1-\cos ^{2} \theta_{3}\right)}{b^{4}}=\frac{a^{2} c^{2}}{b^{4}}\left(1-\frac{\left(a^{2}+b^{2}-c^{2}\right)^{2}}{4 a^{2} b^{2}}\right) \\
& =\frac{c^{2}(a+b+c)(a+b-c)(a+c-b)(b+c-a)}{4 b^{6}} .
\end{aligned}
$$

Since $b+c-a \geqslant a / 100$ and we assumed that $a \geqslant b \geqslant c$, the above quantity is bounded below.

Similarly we have

$$
\begin{aligned}
\left(\frac{\left|u_{1} u_{2}\right| \sin \theta_{2}}{c^{2}}\right)^{2} /\left(\frac{u_{1} u_{2}}{a b}\right)^{2} & =\frac{a^{2} b^{2}\left(1-\cos ^{2} \theta_{2}\right)}{c^{4}}=\frac{a^{2} b^{2}}{c^{4}}\left(1-\frac{\left(a^{2}+c^{2}-b^{2}\right)^{2}}{4 a^{2} c^{2}}\right) \\
& =\frac{b^{2}(a+b+c)(a+b-c)(a+c-b)(b+c-a)}{4 c^{6}}
\end{aligned}
$$

and

$$
\begin{aligned}
\left(\frac{\left|u_{1} u_{3}\right| \sin \theta_{3}}{b^{2}}\right)^{2} /\left(\frac{u_{1} u_{3}}{a c}\right)^{2} & =\frac{a^{2} c^{2}\left(1-\cos ^{2} \theta_{3}\right)}{b^{4}}=\frac{a^{2} c^{2}}{b^{4}}\left(1-\frac{\left(a^{2}+b^{2}-c^{2}\right)^{2}}{4 a^{2} b^{2}}\right) \\
& =\frac{c^{2}(a+b+c)(a+b-c)(a+c-b)(b+c-a)}{4 b^{6}} .
\end{aligned}
$$

They are bounded from below. We proved the theorem in this case.

Case II: $c$ is very small compared with $a$. Now let us look at the case that $c=\mathrm{o}(a)$. Since we already prove the inequality for the case that $b+c-a \geqslant a / 100$, we assume now $b+c-a \leqslant a / 100$. Therefore $c$ is very small compared with both $a$ and $b$.

We consider the minimization problem

$$
\min _{\left(u_{1}, u_{2}, u_{3}\right) \in \mathbb{R}^{3}} \frac{\left|u_{3}\left(u_{1} / b^{2}+\left(u_{2} / a^{2}\right) \cos \theta_{3}\right)\right|+u_{1} u_{2} /(a b)+u_{1} u_{3} /(a c)+u_{2} u_{3} /(b c)}{\left(u_{1}^{2}+u_{2}^{2}\right) / c^{2}+\left(u_{2}^{2}+u_{3}^{2}\right) / a^{2}+\left(u_{1}^{2}+u_{3}^{2}\right) / b^{2}}=J\left(u_{1}, u_{2}, u_{3}\right) .
$$


We consider it as two minimization problems separately,

$$
\min _{\left(u_{1}, u_{2}, u_{3}\right) \in \mathbb{R}^{3}} J_{1}\left(u_{1}, u_{2}, u_{3}\right)=\frac{u_{3}\left(u_{1} / b^{2}+\left(u_{2} / a^{2}\right) \cos \theta_{3}\right)+u_{1} u_{2} /(a b)+u_{1} u_{3} /(a c)+u_{2} u_{3} /(b c)}{\left(u_{1}^{2}+u_{2}^{2}\right) / c^{2}+\left(u_{2}^{2}+u_{3}^{2}\right) / a^{2}+\left(u_{1}^{2}+u_{3}^{2}\right) / b^{2}}
$$

and

$$
\min _{\left(u_{1}, u_{2}, u_{3}\right) \in \mathbb{R}^{3}} J_{2}\left(u_{1}, u_{2}, u_{3}\right)=\frac{-u_{3}\left(u_{1} / b^{2}+\left(u_{2} / a^{2}\right) \cos \theta_{3}\right)+u_{1} u_{2} /(a b)+u_{1} u_{3} /(a c)+u_{2} u_{3} /(b c)}{\left(u_{1}^{2}+u_{2}^{2}\right) / c^{2}+\left(u_{2}^{2}+u_{3}^{2}\right) / a^{2}+\left(u_{1}^{2}+u_{3}^{2}\right) / b^{2}} .
$$

Let

$$
\begin{aligned}
& N=\left|u_{3}\left(\frac{u_{1}}{b^{2}}+\frac{u_{2}}{a^{2}} \cos \theta_{3}\right)\right|+\frac{u_{1} u_{2}}{a b}+\frac{u_{1} u_{3}}{a c}+\frac{u_{2} u_{3}}{b c}, \\
& N_{1}=u_{3}\left(\frac{u_{1}}{b^{2}}+\frac{u_{2}}{a^{2}} \cos \theta_{3}\right)+\frac{u_{1} u_{2}}{a b}+\frac{u_{1} u_{3}}{a c}+\frac{u_{2} u_{3}}{b c}, \\
& N_{2}=-u_{3}\left(\frac{u_{1}}{b^{2}}+\frac{u_{2}}{a^{2}} \cos \theta_{3}\right)+\frac{u_{1} u_{2}}{a b}+\frac{u_{1} u_{3}}{a c}+\frac{u_{2} u_{3}}{b c},
\end{aligned}
$$

and

$$
D=\frac{u_{1}^{2}+u_{2}^{2}}{c^{2}}+\frac{u_{2}^{2}+u_{3}^{2}}{a^{2}}+\frac{u_{1}^{2}+u_{3}^{2}}{b^{2}} .
$$

The minima of $J, J_{1}$ and $J_{2}$ exist on the unit sphere since they are homogeneous. We want to prove that the minimum of $J(u), \theta=N / D \geqslant \max \left(N_{1} / D, N_{2} / D\right)>-1 / 2$, therefore the inequality holds.

At the critical points of $J_{1}$ we have

$$
\begin{aligned}
& \frac{\partial J_{1}}{\partial u_{1}}=\frac{D\left(u_{3} / b^{2}+u_{2} /(a b)+u_{3} /(a c)\right)-N 2 u_{1}\left(1 / b^{2}+1 / c^{2}\right)}{D^{2}}=0, \\
& \frac{\partial J_{1}}{\partial u_{2}}=\frac{D\left(\left(u_{3} / a^{2}\right) \cos \theta_{3}+u_{1} /(a b)+u_{3} /(b c)\right)-N 2 u_{2}\left(1 / a^{2}+1 / c^{2}\right)}{D^{2}}=0, \\
& \frac{\partial J_{1}}{\partial u_{3}}=\frac{D\left(u_{1} / b^{2}+\left(u_{2} / a^{2}\right) \cos \theta_{3}+u_{1} /(a c)+u_{2} /(b c)\right)-N 2 u_{3}\left(1 / a^{2}+1 / b^{2}\right)}{D^{2}}=0 .
\end{aligned}
$$

We must have

$$
\operatorname{det}\left(\begin{array}{ccc}
-2 \theta\left(\frac{1}{b^{2}}+\frac{1}{c^{2}}\right) & \frac{1}{a b} & \frac{1}{b^{2}}+\frac{1}{a c} \\
\frac{1}{a b} & -2 \theta\left(\frac{1}{a^{2}}+\frac{1}{c^{2}}\right) & \frac{1}{a^{2}} \cos \theta_{3}+\frac{1}{b c} \\
\frac{1}{b^{2}}+\frac{1}{a c} & \frac{1}{a^{2}} \cos \theta_{3}+\frac{1}{b c} & -2 \theta\left(\frac{1}{a^{2}}+\frac{1}{b^{2}}\right)
\end{array}\right)=0,
$$

otherwise the only critical point of $J_{1}\left(u_{1}, u_{2}, u_{3}\right)$ would be $(0,0,0)$, which is contradictory with the fact that we are looking for the critical points of $J_{1}\left(u_{1}, u_{2}, u_{3}\right)$ on the unit ball.

Let us look at the coefficients of $1 / c^{4}$,

$$
-(2 \theta)^{3}\left(\frac{1}{a^{2}}+\frac{1}{b^{2}}\right)+2 \theta\left(\frac{1}{a^{2}}+\frac{1}{b^{2}}\right)=F(\theta) .
$$

We must have $F(\theta)=0$ at the critical points of $J_{1}\left(u_{1}, u_{2}, u_{3}\right)$, since $1 / c^{4}$ is the dominant term of the determinant of the linear system. Therefore the minimum of $J_{1}\left(u_{1}, u_{2}, u_{3}\right)$ is either $\theta=0$ or $-1 / 2$.

Similarly, at the critical points of $J_{2}\left(u_{1}, u_{2}, u_{3}\right)$ we have

$$
\begin{aligned}
& \frac{\partial J_{2}}{\partial u_{1}}=\frac{D\left(-u_{3} / b^{2}+u_{2} /(a b)+u_{3} /(a c)\right)-N 2 u_{1}\left(1 / b^{2}+1 / c^{2}\right)}{D^{2}}=0, \\
& \frac{\partial J_{2}}{\partial u_{2}}=\frac{D\left(-\left(u_{3} / a^{2}\right) \cos \theta_{3}+u_{1} /(a b)+u_{3} /(b c)\right)-N 2 u_{2}\left(1 / a^{2}+1 / c^{2}\right)}{D^{2}}=0,
\end{aligned}
$$




$$
\frac{\partial J_{2}}{\partial u_{3}}=\frac{D\left(-u_{1} / b^{2}-\left(u_{2} / a^{2}\right) \cos \theta_{3}+u_{1} /(a c)+u_{2} /(b c)\right)-N 2 u_{3}\left(1 / a^{2}+1 / b^{2}\right)}{D^{2}}=0 .
$$

We must have

$$
\operatorname{det}\left(\begin{array}{ccc}
-2 \theta\left(\frac{1}{b^{2}}+\frac{1}{c^{2}}\right) & \frac{1}{a b} & -\frac{1}{b^{2}}+\frac{1}{a c} \\
\frac{1}{a b} & -2 \theta\left(\frac{1}{a^{2}}+\frac{1}{c^{2}}\right) & -\frac{1}{a^{2}} \cos \theta_{3}+\frac{1}{b c} \\
-\frac{1}{b^{2}}+\frac{1}{a c} & -\frac{1}{a^{2}} \cos \theta_{3}+\frac{1}{b c} & -2 \theta\left(\frac{1}{a^{2}}+\frac{1}{b^{2}}\right)
\end{array}\right)=0 .
$$

The coefficients of $1 / c^{4}$ is

$$
-(2 \theta)^{3}\left(\frac{1}{a^{2}}+\frac{1}{b^{2}}\right)+2 \theta\left(\frac{1}{a^{2}}+\frac{1}{b^{2}}\right)=F_{2}(\theta) .
$$

We must have $F_{2}(\theta)=0$ at critical points, since $1 / c^{4}$ is the dominant term. Therefore the minimum of $J_{2}$ is either $\theta=0$ or $-1 / 2$.

If $J\left(\widetilde{u_{1}}, \widetilde{u_{2}}, \widetilde{u_{3}}\right)=-1 / 2$, then $J_{1}\left(\widetilde{u_{1}}, \widetilde{u_{2}}, \widetilde{u_{3}}\right)=-1 / 2$ and $J_{2}\left(\tilde{u_{1}}, \tilde{u_{2}}, \tilde{u_{3}}\right)=-1 / 2$ must be 0 at the same time. Therefore we have

$$
\left(\begin{array}{ccc}
\left(\frac{1}{b^{2}}+\frac{1}{c^{2}}\right) & \frac{1}{a b} & \frac{1}{b^{2}}+\frac{1}{a c} \\
\frac{1}{a b} & \left(\frac{1}{a^{2}}+\frac{1}{c^{2}}\right) & \frac{1}{a^{2}} \cos \theta_{3}+\frac{1}{b c} \\
\frac{1}{b^{2}}+\frac{1}{a c} & \frac{1}{a^{2}} \cos \theta_{3}+\frac{1}{b c} & \left(\frac{1}{a^{2}}+\frac{1}{b^{2}}\right)
\end{array}\right)\left(\begin{array}{c}
\widetilde{u_{1}} \\
\widetilde{u_{3}} \\
\widetilde{u_{3}}
\end{array}\right)=0,
$$

and

$$
\left(\begin{array}{ccc}
\left(\frac{1}{b^{2}}+\frac{1}{c^{2}}\right) & \frac{1}{a b} & -\frac{1}{b^{2}}+\frac{1}{a c} \\
\frac{1}{a b} & \left(\frac{1}{a^{2}}+\frac{1}{c^{2}}\right) & -\frac{1}{a^{2}} \cos \theta_{3}+\frac{1}{b c} \\
-\frac{1}{b^{2}}+\frac{1}{a c} & -\frac{1}{a^{2}} \cos \theta_{3}+\frac{1}{b c} & \left(\frac{1}{a^{2}}+\frac{1}{b^{2}}\right)
\end{array}\right)\left(\begin{array}{c}
\widetilde{u_{1}} \\
\widetilde{u_{2}} \\
\widetilde{u_{3}}
\end{array}\right)=0 .
$$

Solving it, we get

$$
\tilde{u_{3}}=0 \quad \text { and } \quad \frac{\tilde{u_{1}}}{b^{2}}+\frac{\tilde{u_{2}}}{a^{2}} \cos \theta_{3}=0 .
$$

Therefore

$$
J\left(\widetilde{u_{1}}, \tilde{u_{2}}, \tilde{u_{3}}\right)=\frac{-\left(b / a^{2}\right) \cos \theta_{3}}{\left(1 / c^{2}\right)\left(1+\left(b^{4} / a^{4}\right) \cos ^{2} \theta_{3}\right)+1 / a^{2}+\left(1 / a^{4}\right) \cos ^{2} \theta_{3}} .
$$

Since $c=\mathrm{o}(a), J\left(\widetilde{u_{1}}, \widetilde{u_{2}}, \widetilde{u_{3}}\right)$ is very close to 0 . Thus $J\left(u_{1}, u_{2}, u_{3}\right)$ can never reach $-1 / 2$.

We proved the theorem in this case.

Case III: all the remaining cases. The only case left is the case that $c / a \geqslant \gamma$ and $b+c-a \leqslant a / 100$, here $\gamma$ is a fixed small number. In this case, the lengths of $a, b$ and $c$ are comparable.

Since in this case $\sin ^{2} \theta_{1}=1-\left(b^{2}+c^{2}-a^{2}\right)^{2} /(2 b c)^{2}$ is very close to 0 , and $a$ is the largest side of the triangle, we know that $\theta_{1}$ is very close to $\pi$. On the other hand, $\sin ^{2} \theta_{2}=1-\left(a^{2}+c^{2}-b^{2}\right)^{2} /(2 a c)^{2}$ and $\sin ^{2} \theta_{3}=1-$ $\left(b^{2}+a^{2}-c^{2}\right)^{2} /(2 a b)^{2}$ are also very small, therefore $\theta_{2}$ and $\theta_{3}$ are very close to 0 . Thus we have

$$
\begin{aligned}
& \left|u_{1}\right| \sqrt{\frac{u_{2}^{2}}{c^{4}}+\frac{u_{3}^{2}}{b^{4}}+2 u_{2} u_{3} \frac{\cos \theta_{1}}{b^{2} c^{2}}} \geqslant\left|\frac{u_{1} u_{2}}{c^{2}}-\bar{\gamma} \frac{u_{1} u_{3}}{b^{2}}\right|, \\
& \left|u_{2}\right| \sqrt{\frac{u_{1}^{2}}{c^{4}}+\frac{u_{3}^{2}}{a^{4}}+2 u_{1} u_{3} \frac{\cos \theta_{2}}{a^{2} c^{2}}} \geqslant\left|\frac{u_{1} u_{2}}{c^{2}}+\bar{\gamma} \frac{u_{2} u_{3}}{a^{2}}\right|,
\end{aligned}
$$


and

$$
\left|u_{3}\right| \sqrt{\frac{u_{1}^{2}}{b^{4}}+\frac{u_{2}^{2}}{a^{4}}+2 u_{1} u_{2} \frac{\cos \theta_{3}}{a^{2} b^{2}}} \geqslant\left|\frac{u_{1} u_{3}}{b^{2}}+\bar{\gamma} \frac{u_{2} u_{3}}{a^{2}}\right|,
$$

here $\bar{\gamma}$ is almost 1 .

Subcase $i: u_{2} u_{3}<0$. If $u_{2} u_{3}<0$, then we have

$$
\left|\frac{u_{1} u_{2}}{c^{2}}-\bar{\gamma} \frac{u_{1} u_{3}}{b^{2}}\right|=\frac{\left|u_{1} u_{2}\right|}{c^{2}}+\bar{\gamma} \frac{\left|u_{1} u_{3}\right|}{b^{2}},
$$

and either

$$
\left|\frac{u_{1} u_{2}}{c^{2}}+\bar{\gamma} \frac{u_{2} u_{3}}{a^{2}}\right|=\frac{\left|u_{1} u_{2}\right|}{c^{2}}+\bar{\gamma} \frac{\left|u_{2} u_{3}\right|}{a^{2}}
$$

or

$$
\left|\frac{u_{1} u_{3}}{b^{2}}+\bar{\gamma} \frac{u_{2} u_{3}}{a^{2}}\right|=\frac{\left|u_{1} u_{3}\right|}{b^{2}}+\bar{\gamma} \frac{\left|u_{2} u_{3}\right|}{a^{2}} .
$$

Comparing with $u_{1} u_{2} /(a b)+u_{1} u_{3} /(a c)+u_{2} u_{3} /(b c)$, since the lengths of $a, b$ and $c$ are comparable in this case, we can find a constant $\tilde{c}$ such that

$$
\left|\frac{u_{1} u_{2}}{c^{2}}-\bar{\gamma} \frac{u_{1} u_{3}}{b^{2}}\right|+\left|\frac{u_{1} u_{2}}{c^{2}}+\bar{\gamma} \frac{u_{2} u_{3}}{a^{2}}\right|+\left|\frac{u_{1} u_{3}}{b^{2}}+\bar{\gamma} \frac{u_{2} u_{3}}{a^{2}}\right| \geqslant \tilde{c}\left(\frac{u_{1} u_{2}}{a b}+\frac{u_{1} u_{3}}{a c}+\frac{u_{2} u_{3}}{b c}\right) .
$$

We proved the theorem in this subcase.

Subcase ii: $u_{2} u_{3}>0$ and $u_{1} u_{2} u_{3}<0$. If $u_{1}, u_{2}$ and $u_{3}$ are all negative, the theorem is trivial. Therefore we need only to consider the case that $u_{1}<0$ and $u_{2}, u_{3}>0$. We need to prove that there exist $\theta$ such that

$$
\begin{aligned}
\max \left\{\left|\frac{u_{1} u_{2}}{c^{2}}-\bar{\gamma} \frac{u_{1} u_{3}}{b^{2}}\right|,\left|\frac{u_{1} u_{2}}{c^{2}}+\bar{\gamma} \frac{u_{2} u_{3}}{a^{2}}\right|,\left|\frac{u_{1} u_{3}}{b^{2}}+\bar{\gamma} \frac{u_{2} u_{3}}{a^{2}}\right|\right\} \\
+\left(\frac{u_{1}}{c}+\frac{u_{3}}{a}\right)^{2}+\left(\frac{u_{2}}{c}+\frac{u_{3}}{b}\right)^{2}+\left(\frac{u_{1}}{b}+\frac{u_{2}}{a}\right)^{2} \\
\geqslant \theta\left(\frac{u_{1}^{2}+u_{2}^{2}}{c^{2}}+\frac{u_{2}^{2}+u_{3}^{2}}{a^{2}}+\frac{u_{1}^{2}+u_{3}^{2}}{b^{2}}\right) .
\end{aligned}
$$

The inequality is true if either $\left(u_{1} / c+u_{3} / a\right)^{2} \geqslant \tilde{\gamma}\left(u_{1} / c\right)^{2}$ or $\left(u_{1} / b+u_{2} / a\right)^{2} \geqslant \tilde{\gamma}\left(u_{1} / c\right)^{2}$ holds, here $\tilde{\gamma}$ is a fixed small constant. Hence we only need to explore the case when $\frac{a}{c}(-\tilde{\gamma}-1) u_{1} \leqslant u_{3} \leqslant \frac{a}{c}(\tilde{\gamma}-1) u_{1}$ and $\frac{a}{b}(-\tilde{\gamma}-1) u_{1} \leqslant$ $u_{2} \leqslant \frac{a}{b}(\tilde{\gamma}-1) u_{1}$. Since we assume $u_{1}<0$ and $u_{2}, u_{3}>0$, this can never happen.

Subcase iii: $u_{2} u_{3}>0$ and $u_{1} u_{2} u_{3}>0$. If $u_{1}, u_{2}$ and $u_{3}$ are all positive, the theorem is trivial. Therefore we need only to consider the case that $u_{1}>0$ and $u_{2}, u_{3}<0$.

The theorem holds if either $\left(u_{1} / c+u_{3} / a\right)^{2} \geqslant \gamma\left(u_{1} / c\right)^{2}$ or $\left(u_{1} / b+u_{2} / a\right)^{2} \geqslant \gamma\left(u_{1} / c\right)^{2}$, here $\gamma$ is a small fixed constant. Therefore we only need to explore the case when $\frac{a}{c}(-\gamma-1) u_{1} \leqslant u_{3} \leqslant \frac{a}{c}(\gamma-1) u_{1}$ and $\frac{a}{b}(-\gamma-1) u_{1} \leqslant$ $u_{2} \leqslant \frac{a}{b}(\gamma-1) u_{1}$. Under this condition,

$$
\left|\frac{u_{1} u_{2}}{c^{2}}+\frac{u_{2} u_{3}}{a^{2}}\right| \approx\left|\frac{a}{b}(\gamma-1) \frac{1}{c^{2}}+\frac{(\gamma-1)^{2}}{b c}\right| u_{1}^{2} \approx\left|\frac{1}{b c}-\frac{a}{b} \frac{1}{c^{2}}\right| u_{1}^{2}=\frac{1}{b c}\left|1-\frac{a}{c}\right| u_{1}^{2} .
$$

Since $b+c-a \leqslant a / 100$, we have $a / c>200 / 101$.

Therefore

$$
\left|\frac{u_{1} u_{2}}{c^{2}}+\frac{u_{2} u_{3}}{a^{2}}\right| \geqslant \theta u_{1}^{2} \text {. }
$$

Since $u_{2} u_{3}>0$ and the length of $a, b$ and $c$ are comparable, the $u_{2}^{2}$ and $u_{3}^{2}$ terms have been taken care of.

Thus we established the theorem, i.e. the inequality in the case of three masses. 


\section{Heuristic argument for general $p$}

After providing a clear proof for $p=3$, we present here a heuristic argument which shows why such an inequality should hold. We believe that this inequality should give rise to a continuous (maybe well-known) inequality after proper continuation.

We want to show in this section that condition $(\mathrm{H})$ on the eigenvalues of $A$ implies the theorem. Unfortunately, at this moment we do know how to prove this condition on the eigenvalues. $\rho$ is an eigenvalue of $A$. The condition on $\rho$ reads,

(H) Assume $\rho=0$, then $\nabla \rho \neq 0$.

We show then why the inequality might be true if $(\mathrm{H})$ holds. We consider it in two cases, the first case when the distance between all the points $x_{i}$ 's are comparable, and the case that some of the distances are very small, and some are huge.

Case 1. The distance between all the points $x_{i}$ 's is comparable, i.e. there exists a constant $C$ such that,

$$
\frac{1}{C} \leqslant \frac{\left|x_{i}-x_{j}\right|}{\left|x_{l}-x_{s}\right|} \leqslant C
$$

Then either all eigenvalues are greater than $\theta$, which is fixed; or there exists some eigenvalue $\rho$ very small, then thanks to condition $(\mathrm{H}), \nabla \rho \neq 0$ in this case.

Case 1(a). All eigenvalues are greater than $\theta$. It is easy to see for all $u$,

$$
|A u| \geqslant \theta|u| \text {. }
$$

The inequality follows.

Case 1(b). There exists some eigenvalue $\rho$ very small. Assume $u$ is the unit eigenvector corresponding to $\rho$, then

$$
\left|{ }^{t} u \frac{\partial A}{\partial x_{i}^{j}} u\right|=\left|\frac{\partial}{\partial x_{i}^{j}}\left({ }^{t} u A u\right)-2^{t} u A \frac{\partial u}{\partial x_{i}^{j}}\right|=\left|\frac{\partial}{\partial x_{i}^{j}} \rho-2 \rho^{t} u \frac{\partial u}{\partial x_{i}^{j}}\right| .
$$

Since ${ }^{t} u u=1$, we have ${ }^{t} u \partial u / \partial x_{i}^{j}=0$. Therefore,

$$
\left|{ }^{t} u \frac{\partial A}{\partial x_{i}^{j}} u\right|=\left|\frac{\partial}{\partial x_{i}^{j}} \rho\right| \geqslant \theta_{i}>0 .
$$

The inequality follows if only one eigenvalue is small.

Case 2. Some of the $\left|x_{i}-x_{j}\right| /\left|x_{l}-x_{s}\right|$ can become very large or very small.

Assume $x_{2} \cdots x_{l}$ are very close to $x_{1}$, i.e. $\left|x_{j}-x_{1}\right| \leqslant \varepsilon$, and the remaining points $x_{k+1}, \ldots, x_{k}$ are such that $\varepsilon \leqslant\left|x_{j}-x_{1}\right| \leqslant 1$. The matrix $A$ reads

$$
\left(\begin{array}{cc}
B & C \\
C^{t} & D
\end{array}\right)
$$

For the upper matrix $B$, since the distance between any two points of $x_{1}, \ldots, x_{l}$ is very small, each element of $B$ is greater than $1 / \varepsilon$; But each element of $C$ is between 1 and $1 / \varepsilon$, since $1 \leqslant\left|x_{i}-x_{j}\right| \leqslant 1 / \varepsilon$. If we scale $A$ such that the largest element is 1 , then all the elements of $C$ become very small. Thus we can think $A$ as the direct sum of the two sub-matrices $B$ and $D$. We can prove the inequality using induction then.

\section{References}

[1] M.O. Ahmedou, K.O. El Mehdi, Computation of the difference of topology at infinity for Yamabe-type problems on annuli-domains. I, II, Duke Math. J. 94 (2) (1998) 215-229, 231-255.

[2] A. Bahri, Critical Points at Infinity in Some Variational Problems, Pitman Res. Notes Math. Ser., vol. 182, Longman Scientific \& Technical, Harlow, 1989.

[3] A. Bahri, An invariant for Yamabe-type flows with applications to scalar-curvature problems in high dimension, A celebration of John F. Nash, Jr., Duke Math. J. 81 (2) (1996) 323-466. 
[4] A. Bahri, H. Brezis, Non-linear elliptic equations on Riemannian manifolds with the Sobolev critical exponent, in: Topics in Geometry, in: Progr. Nonlinear Differential Equations Appl., vol. 20, Birkhäuser Boston, Boston, MA, 1996, pp. 1-100.

[5] A. Bahri, S. Chanillo, The difference of topology at infinity in changing-sign Yamabe problems on $S^{3}$ (the case of two masses), Comm. Pure Appl. Math. 54 (4) (2001) 450-478.

[6] A. Bahri, J.-M. Coron, On a nonlinear elliptic equation involving the critical Sobolev exponent: the effect of the topology of the domain, Comm. Pure Appl. Math. 41 (3) (1988) 253-294.

[7] A. Bahri, Y. Li, O. Rey, On a variational problem with lack of compactness: the topological effect of the critical points at infinity, Calc. Var. Partial Differential Equations 3 (1) (1995) 67-93.

[8] A. Bahri, Y. Xu, Recent progress in conformal and contact geometry, in press.

\section{Further reading}

[9] M. Struwe, A global compactness result for elliptic boundary value problems involving limiting nonlinearities, Math. Z. 187 (4) (1984) 511517. 